\title{
Doppler Radar Sensing of Multiple Subjects in Single and Multiple Antenna Systems
}

\author{
Olga Boric-Lubecke ${ }^{1}$, Victor M. Lubecke ${ }^{1}$, Anders Host-Madsen ${ }^{1}$, \\ Dragan Samardzija ${ }^{2}$, and Ken Cheung ${ }^{3}$
}

\begin{abstract}
Doppler radar life sensing has shown promise in medical and security applications, however the problems of motion artifacts and presence of multiple subjects limit the usefulness of this technique. By leveraging recent advances in signal processing and wireless communications technologies, the Doppler radar technique has the potential to overcome these limitations. We will explore the single and multiple antenna systems and SIMO/MIMO signal processing to isolate desired radar return signals from multiple subjects. It has been experimentally demonstrated that up to two subjects can be separated in a single antenna systems. Simulations have also shown that in case two subjects have identical cardiovascular behavior, it is possible to distinguish them using MIMO techniques.
\end{abstract}

Keywords - Remote life sensing, detection of heart and respiration activity, multiple antenna systems, Doppler radar, multiple subjects.

\section{INTRODUCTION}

Doppler radar remote detection of heart and respiration activity is a promising technique for unobtrusive health monitoring and life sensing, with proof of concept demonstrated for various applications [1-3]. However, so far this approach has been limited to sensing and detection of a single subject. When there are two or more subjects present in the environment, it is more challenging to isolate signals from individual subjects. We are exploring separation of signals from multiple subjects in a single and multiple antenna systems. In a single antenna system, based on expected different spectral signatures of individual cardiovascular related motion, it is possible to separate individual signals in frequency domain. In a multiple antenna system (SIMO or MIMO), even if individual cardiovascular signatures are very similar, it is possible to distinguish different subjects based on angle of arrival. In this paper we will discuss theoretical

\footnotetext{
${ }^{1}$ O. Boric-Lubecke, Anders Host-Madsen, and V. M. Lubecke are with the University of Hawai'i at Manoa, Honolulu, Hawaii, 96822 USA.

${ }^{2}$ Dragan Samardzija is with Bell Laboratories, Lucent Technologies, L-273, 791 Holmdel-Keyport Road, Holmdel, NJ 07733, USA.

${ }^{3}$ Ken Cheung is with Oceanit, 1001Bishop St, ASB Tower, Suite 2970, Honolulu HI 96813, USA.
}

background for both of these approaches, experimental results for a single antenna system, and simulation results for the MIMO system.

Single Doppler radar antenna systems have been described in [4-8]. In these systems, analog signal conditioning was used to amplify baseband output, cut-off the DC component, and avoid aliasing, while digital signal processing was used to separate breathing and heart signals from a single subject, and determine heart and breathing rates. In a single antenna system described here digital signal processing is additionally used to identify spectral signatures from multiple subjects based on FFT approach.

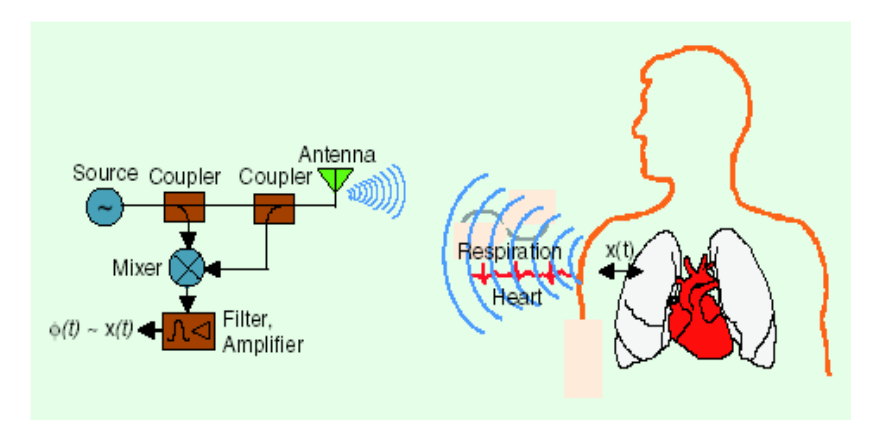

Fig. 1 A single antenna system with a single subject. A homodyne radio is used to detect a phase shift proportional to chest displacement due to cardiopulmonary acitivy.

The multiple antenna approach is based on recent developments in multiple input multiple output (MIMO) signal processing techniques. Lucent Technologies has been the leader in this field with Bell-Labs LAyered Space-Time (BLAST) techniques [9]. We propose to use techniques based on BLAST to separate useful scattered radio signals from multiple individuals. When more than one target is in view, multiple transmitters and receivers providing multiple signal copies could be used to distinguish between the different sources of Doppler motion, isolate the desired signal, and determine a number of targets. In this paper, we present MIMO system simulation results that indicate that it is possible to separate multiple subjects even with identical breathing and heart rates. 


\section{Single ANTENNA SySTEMS}

A single antenna system, with one subject in the antenna field of view, is illustrated in Fig. 1. The return signal is mixed with a sample of the transmitted signal to produce an output voltage with its magnitude proportional to the phase

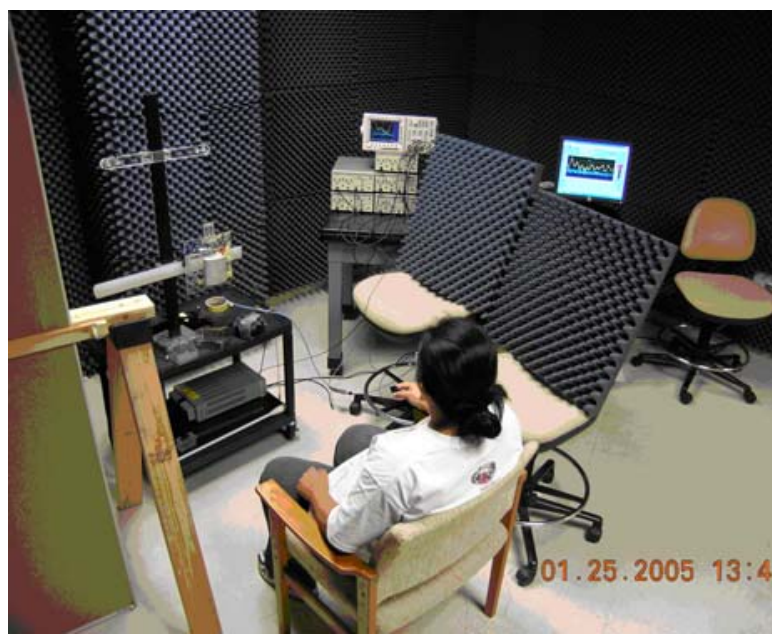

(a)

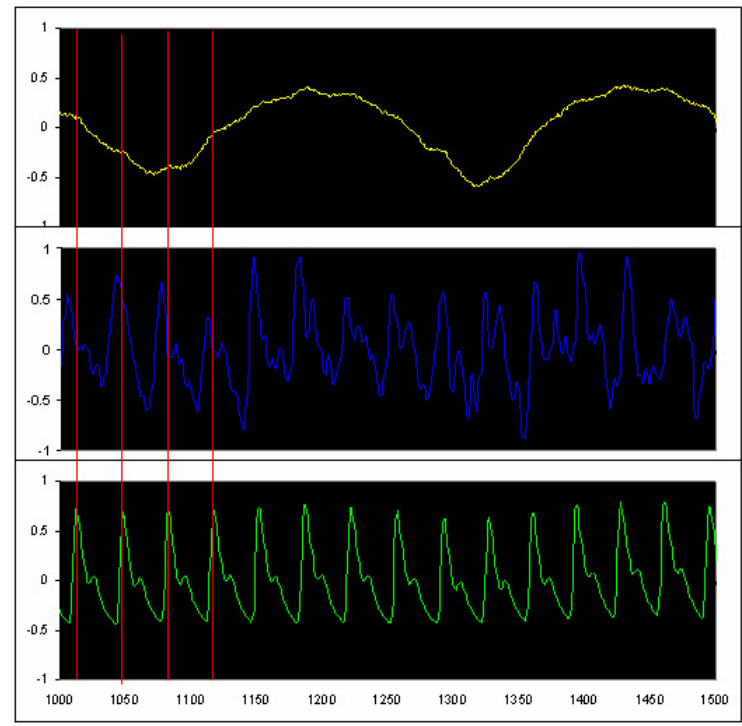

(b)

Fig. 2. Photograph of a single antenna Doppler radar set-up (a), and output signals (b). Cardiopulmonary signals were obtained for a subject seated at about 1 meter away from the antenna. Top trace shows Doppler output filtered to contain breathing and heart signals, middle trace heart only, and bottom trace shows the finger pressure pulse reference taken to evaluate the accuracy of the measurements.

shift between them, which in turn is proportional to chest displacement due to cardiopulmonary activity. Doppler radar was assembled using following commercially available components: Agilent CW signal source (HP E4433B ESG-D series), Mini-Circuits ZFM-4212 mixer and ZFSC-2-2500 coupler, Narda 4923 circulator, and Antenna Specialist patch antenna ASPPT 2988, with $60^{\circ}$ by $80^{\circ}$ beamwidth. Circulator was used at the antenna connector to provide better isolation between transmit and receive signals. Baseband analog signal conditioning was performed using Stanford Research Systems Model SR560 low noise amplifiers/filters. The filter output was then digitized using a 12-bit analog to digital converter, and data was further processed in digital domain. A finger pressure pulse reference, taken with UFI-1010 pulse

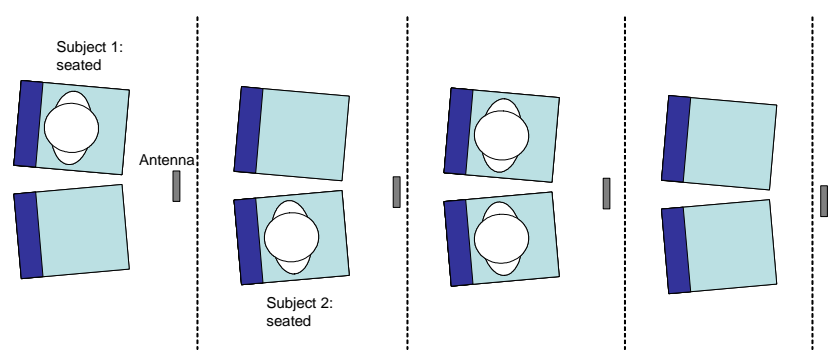

(a)

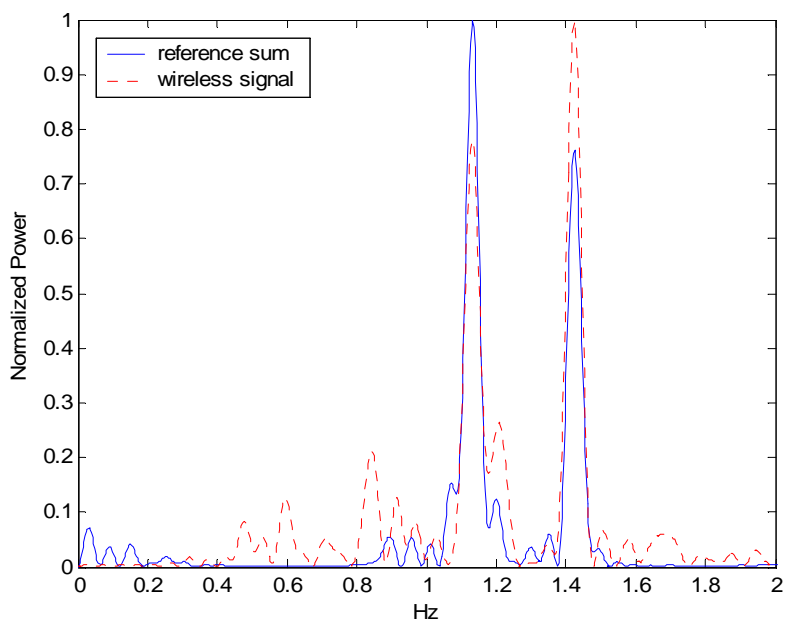

(b)

Fig. 3. The measurement set-up for single antenna radar system with two subjects (a), and output heart signals in frequency domain (b). Two distinct heart rates can be clearly identified (b), with a good agreement with the reference signals.

transducer, was used to evaluate the measurement accuracy. All radar measurements were conducted with the CW signal source at $2.4 \mathrm{GHz}$ with $0 \mathrm{dBm}$ output power. Mixer output analog signal conditioning was performed with a high pass filter with $0.03 \mathrm{~Hz}$ cut-off and slope of $18 \mathrm{~dB} /$ octave followed by a low pass filter with $30 \mathrm{~Hz}$ cut-off, slope of $6 \mathrm{~dB} /$ octave and gain of about 100. Additional high pass filter with $1 \mathrm{~Hz}$ cut-off, slope of $12 \mathrm{~dB} /$ octave, and gain of 2 was used to isolate heart signal only. 


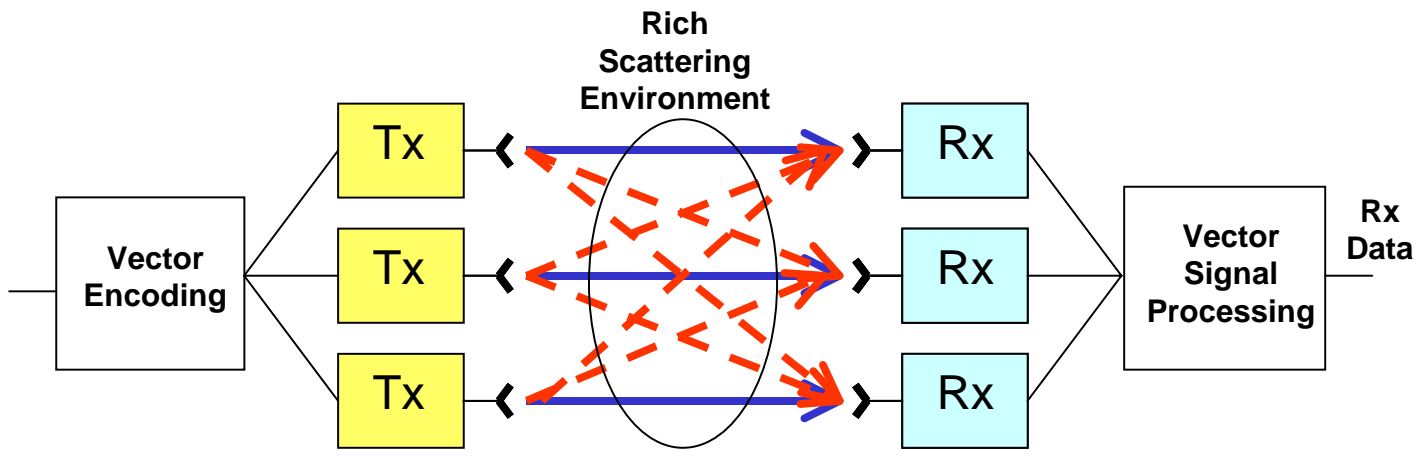

Fig. 4. Multiple input multiple output (MIMO) system overview. Capacity for a wireless communications channel can be increased by using multipath scattering to create independent parallel subchannels that can be coherently combined. While each receiver $(\mathrm{Rx})$ receives a linear combination of all transmitted $(\mathrm{Tx})$ components, sophisticated signal processing can be used to isolate and recover these components through a priori knowledge of their make up and characterization of the channel environment.

A reference signal was bandpass filtered in the frequency range of $0.03-30 \mathrm{~Hz}$, with a slope of $6 \mathrm{~dB} /$ octave. Figure 2 shows a photograph of a single antenna Doppler radar set-up (a), and output signals in time domain (b), where horizontal axes indicates the number of samples. Cardiopulmonary signals were obtained for a subject seated at about 1 meter away from the antenna. Top trace in Fig. 2 (b) shows Doppler radar output filtered to contain breathing and heart signals, while middle trace was further band-pass filtered for heart signal only. The bottom trace shows the finger pressure pulse reference, with signal peaks clearly corresponding to the heart signal peaks in the middle trace.

A greater challenge was to use the radar to measure more than one target. We used a single radar transceiver to measure signals from up to two stationary targets. The experimental setup is shown in Fig. 3 (a). The possible subject combinations are as follows:

(1) Single subject in room, seated in first chair, connected to first reference sensor;

(2) Different single subject in room, seated in second chair, connected to second reference sensor;

(3) Both subjects seated in chairs, connected to respective reference sensors;

(4) No subjects in room.

For this simple controlled case, we chose two subjects with different resting heart rates. Using the single transceiver, data were taken for all cased described above and analyzed using a fast Fourier transform (FFT). Figure 3 (b) shows the FFT of a 20 -second segment data taken for the case (3) with both subjects seated at about the same distance from the antenna. The red, dashed curve (Fig. 3(b)) is the FFT of radar signal, and the blue curve is the FFT of sum of the two reference signals, taken for comparison. Two distinct heart rates can be clearly identified in Fig. 3 (b), with a good agreement with the reference signals. This simple test showed that it was possible to measure and distinguish two subjects using a single transceiver without sophisticated processing schemes.

\section{MultiPle ANTENNA Systems}

A multiple input multiple output (MIMO) system configuration, illustrated in Fig. 4, can be used in wireless communications to enhance channel capacity. Such a system takes advantage of random scattering of radio signals between transmit and receive antennas. This scattering is known as multipath, since it results in multiple copies of the transmitted signal arriving at the receivers via different scattered paths. In conventional wireless systems, multipath could result in destructive interference, and is thus considered undesirable. However, MIMO systems exploit multipath to enhance transmission accuracy by treating scattering paths as separate parallel subchannels. In a BLAST implementation of a MIMO system [9], a single user's data stream is split into multiple substreams, using orthogonal codes in the same frequency band, and each transmitter (Tx) antenna transmits one such substream. At the other end, each receiver $(\mathrm{Rx})$ antenna receives a linear combination of all transmitted substreams. As a result of multipath these combinations are slightly different at each antenna.

We propose to use ideas and technologies from MIMO systems for wireless communications to enhance the quality of Doppler radar cardiopulmonary signals, and to separate signals originating from multiple sources. If there is a single source (one person), multiple receive antennas can be used to increase the SNR of the signal, and separate the desired signal 
from interference. With multiple sources, the system can be used to separate content from the different sources at the receiving end using techniques from blind source separation (BSS) [10]. In this case, some characteristics of the desired signals are known and this information can be used to help

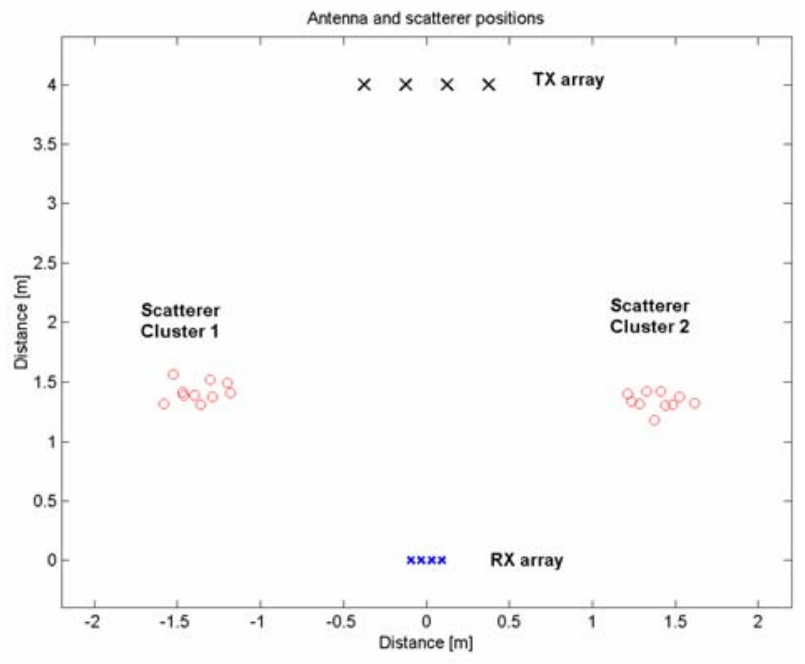

(a)

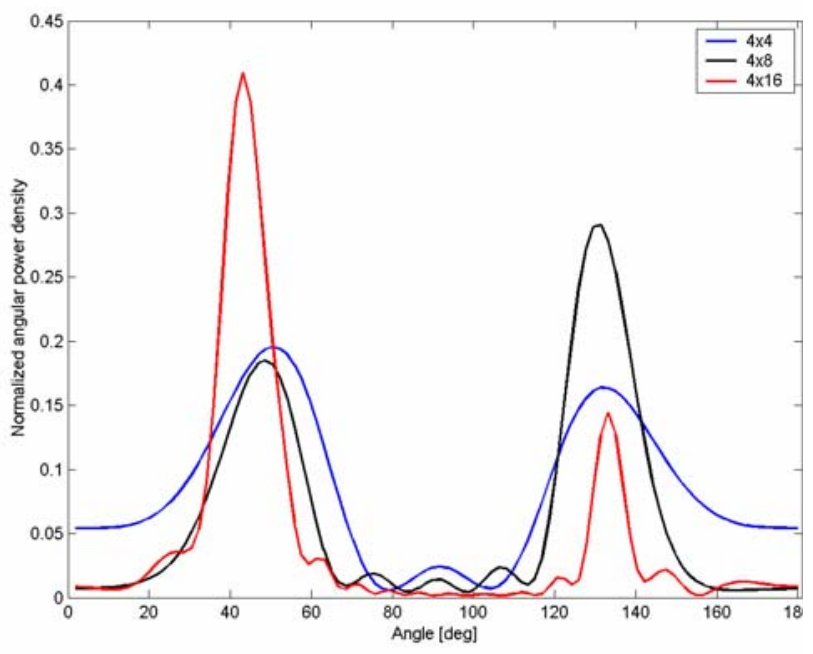

(b)

Fig. 5. An arrangement of $\mathrm{Tx}$ and $\mathrm{Rx}$ antennas, and two clusters of scatterers (a), and the normalized angular power density for different number of $\mathrm{Rx}$ antennas and two individuals (b). Two dominant directions of arrival at angles $45^{\circ}$ and $135^{\circ}$ (corresponding to the cluster 1 and 2, respectively) can be clearly identified, and as the number of Rx antenna increases the maximums are becoming narrower allowing for more accurate estimation of the direction of arrival.

separate the sources. To be able to implement BSS algorithms for Doppler radar detection of heart and respiration signals, we first need to develop the models for the types of signals that we will be observing. Since the origin of the Doppler signals is well localized in space (person's chest), we expect a small angular spread of 1 to 10 degrees in received signals. To model the cardiopulmonary activity we assume that the position of chest surface is oscillating in time on two different frequencies with different amplitudes. The first frequency and amplitude model the cardio activity (typical frequency is $1 \mathrm{~Hz}$ and the amplitude is $0.1 \mathrm{~mm}$ ), while the second frequency and amplitude model the pulmonary activity (typical frequency is $0.3 \mathrm{~Hz}$ and the amplitude is $10 \mathrm{~mm}$ ) [11-12].

A MIMO system requires extensive signal processing to recover the original signal from received copies. The signal received at multiple Rx antennas (the multiple outputs) will be sampled at a high rate and multiplexed in time, forming a large database of space-time samples. Using previously built models, this database will be searched for familiar patterns. Once a pattern is recognized, it will be removed from the signal combinations, and the search will continue until all signals are recovered.

While Single Input Multiple Output (SIMO) systems in wireless communications can provide diversity gain, array gain, and interference canceling gain, they are still providing only one source signal. In the case of Doppler radar, even if we have a single Tx antenna, we will essentially have as many independent signals as there are scatterers, since the subject and objects in subject's vicinity will scatter radio waves (acting as secondary sources) resulting in independent phase shifts. If we use $\mathrm{N} \mathrm{Rx}$ antennas, we will obtain $\mathrm{N}$ linear combinations of scattered signals. We can also use multiple Tx and Rx antennas in a Doppler radar system. Similarly to wireless systems, where $\mathrm{Tx}$ antennas can be identified by orthogonal codes, in a Doppler radar system each antenna could transmit a signal at a slightly different frequency to simplify channel estimation.

Simulations were done for a MIMO system with 4 transmit and 4 receive antennas, with the carrier frequency of 2.4 $\mathrm{GHz}$, and $2 \lambda$ and $\lambda / 2$ separation between transmit and receive antenna elements, respectively (where $\lambda$ is the wavelength) [13]. The two arrays were assumed to be 4 meters apart. The subject is modeled as a randomly positioned scatterer cluster of 10 scatterers, with the diameter $\mathrm{D}=0.5 \mathrm{~m}$. Note that the effective area of each isotropic scatterer is selected with the assumption that half of the incident energy on the human body is reflected [14]. Receive antennas are assumed to be isotropic with $0 \mathrm{~dB}$ gain. To model the cardiopulmonary activity the position of each scatterer, with respect to the cluster center, is oscillating in time on two different frequencies with different amplitudes as discussed above. To analyze temporal variations in the MIMO channel, we collected a sequence of the channel state estimates. To illustrate potentials of the multiple antenna systems to exploit spatial aspects, we observe a case of two individuals with same frequency and amplitude of the cardiopulmonary activity at different locations in the room that are not a priori known to the receiver. After performing the temporal spectrum analysis, it is not possible to distinguish multiple subjects. In order to estimate spatial characteristics of specific 
spectral components of the channel state estimate sequence, we perform the following steps:

(1) Determine the power spectrum of the channel state estimate sequence averaged over all Tx-Rx pairs;

(2) Select a frequency component of interest (e.g., breathing component). For individual Tx-Rx pair (there is total of $n_{t x} \mathrm{X}$ $n_{r x}$ pairs), perform the Fourier transformation and select the coefficient corresponding to the desired frequency component;

(3) Determine how much power is arriving to the Rx antenna array from the direction corresponding to angles from 0 to 180 degrees.

Figure 5 shows the arrangement of $\mathrm{Tx}$ and $\mathrm{Rx}$ antennas, and two clusters of scatterers (a), and the normalized angular power density for different number of Rx antennas for the individuals located as given in Fig. 5 (a). The results are presented for $n_{t x}=4$ and the different number of Rx antennas $\left(n_{r x}=4,8\right.$ and 16). Two dominant directions of arrival can be clearly identified at angles $45^{\circ}$ and $135^{\circ}$ (Fig. 5 (b)), corresponding to the cluster 1 and cluster 2 , respectively. We also note that as the number of $\mathrm{Rx}$ antenna increases the peaks of the normalized angular spectral density are becoming narrower allowing for more accurate estimation of the direction of arrival. Similar results can be obtained if the number of Tx antennas is varied. These results indicate that it is possible to distinguish multiple subjects with the same heart and breathing rates based on angle of arrival. Combining this technique with the frequency method applied in single antenna systems might enable resolution of a higher number of subjects than with either technique alone.

\section{CONCLUSIONS}

Remote life sensing could be a powerful tool in applications where it is not desirable to disturb a subject's physiological and/or emotional state during detection or in other situations where access to the subject is limited. Although microwave Doppler radar sensing has shown promise toward this goal, it has not suited practical applications in remote subject detection due to the difficulty in distinguishing useful signals resulting from heart and respiration activity from signals produced by environmental motion, and the presence of other people. We believe advances could be made for this technology through a research effort focused on separating signals from multiple subjects through frequency and spatial techniques. Multiantenna systems have led to dramatic improvements in wireless channel capacity. We believe that through the development of similar and related techniques for Doppler radar, it will be possible to overcome the current fundamental problems of useful signal loss due to random motion and interference from other subjects within radar range. Such techniques would also enable us to determine a number of subjects as well as monitor several subjects simultaneously. It has been experimentally demonstrated that up to two subjects can be separated in a single antenna systems, assuming that the subjects have different resting heart rates. Simulations have also shown that in case two subjects have identical cardiovascular behavior, it is possible to distinguish them using MIMO techniques based on angle of arrival. Future research with include experimental implementation of a Doppler radar MIMO system, as well as algorithm development for determining a number of subject in the observed area.

\section{ACKNOWLEDGEMENTS}

The authors would like to thank Paul Pernambuco-Wise for software implementation, and Wansuree Massagram, ByongKwon Park, Shuhei Yamada, Ivy Lo, Mingqi Chen, Xiaoyue Wang, and Daniel Ling for their help with data collection. The authors gratefully acknowledge the support of US Army RDECOM CERDEC I2WD under contract W15P7T-05-CB202. This work was partially supported by NSF grant \# ECS04-28975.

\section{REFERENCES}

[1] J. C. Lin, "Microwave sensing of physiological movement and volume change: a review," Bioelectromagnetics, vol. 13, pp. 557-565, 1992.

[2] K. M. Chen, D. Mirsa, H. Wang, H. R. Chuang, E. Postow, “An X-band microwave life detection system,” IEEE Transactions of Biomedical Eng., vol. 33, pp. 697-70, 1986.

[3] V.M. Lubecke, O. Boric-Lubecke, G. Awater, P.-W. Ong, P. Gammel, R.-H. Yan, J.C. Lin, "Remote sensing of vital signs with telecommunications signals,” World Congress on Medical Physics and Biomedical Engineering (WC2000), Chicago IL, July 2000.

[4] A. D. Droitcour, V. M. Lubecke, J. Lin and O. Boric-Lubecke, "A Microwave Radio for Doppler Radar Sensing of Vital Signs,” 2001 IEEE MTT-S IMS Digest, vol. 1, pp. 175-178, 2001.

[5] A.D. Droitcour, O. Boric-Lubecke, V. M. Lubecke and J. Lin, “0.25 $\mu \mathrm{m}$ CMOS and BiCMOS Single-chip Direct-conversion Doppler Radars for Remote Sensing of Vital Signs,” 2002 ISSCC Digest, vol.1 pp.348-348, 2002.

[6] V. M. Lubecke, O. Boric-Lubecke, and E. Beck, "A Compact Low-Cost Add-On Module for Doppler Radar Sensing of Vital Signs Using a Wireless Communications Terminal,” 2002 IEEE MTT-S IMS Digest, 2002.

[7] B. Lohman, O. Boric-Lubecke, V. M. Lubecke, P. W. Ong, and M. M. Sondhi, “A digital signal processor for Doppler radar sensing of vital signs," IEEE Engineering in Medicine and Biology Conf., Istanbul, Turkey, October 2001.

[8] O. Boric-Lubecke, A. D. Droitcour, V. M. Lubecke, J. Lin, and G. T. A. Kovacs, "Wireless IC Doppler Radars for Sensing of Heart and Respiration Activity," 6th International Conference on Telecommunications in Modern Satellite, Cable and Broadcasting Services (TELSIKS 2003) Nis, Serbia and Montenegro, October 2003. 
[9] G. J. Foschini, "Layered Space-Time Architecture for Wireless Communication in a Fading Environment When Using Multiple Antennas,” Bell Labs Technical Journal, Vol. 1, No. 2, Autumn 1996, pp 41-59.

[10] C Cardoso, "Blind signal separation: statistical principles", Proceedings of the IEEE, Vol. 86, No. 10, October 1998.

[11] T. Kondo, T. Uhlig, P. Pemberton, P. D. Sly, "Laser Monitoring of Chest Wall Displacement,” Eur. Respir. Journal, Vol. 10, pp. 1865-1869, 1997.

[12] G. Ramachandran, S. Swarnamani, and M. Singh, "Reconstruction of Out-of-Plane Cardiac Displacement Patterns as Observed on the Chest Wall During Various Phases of ECG by Capacitance Transducer," IEEE Trans. on Biomed. Eng., Vol. 38, No. 4 pp. 383-385, 1991.

[13] D. Samardzija, O. Boric-Lubecke, A. Host-Madsen, V. M. Lubecke, T. Sizer, A. D. Droitcour and G. T. A. Kovacs, "Applications of MIMO Techniques to Sensing of Cardiopulmonary Activity", IEEE/ACES International Conference on Wireless Communications and Applied Computational Electromagnetics, 2005.

[14] J. C. Lin, "Microwave Propagation in Biological Dielectrics with Application to Cardiopulmonary Interrogation," IEEE Press, Medical Applications of Microwave Imaging, pp. 47-48, NY, 1986. 